\title{
Análise espacial da criminalidade no Rio Grande do Sul
}

\author{
Cristiano Aguiar de Oliveira
}

Resumo: Este artigo faz uma análise espacial da criminalidade no Estado do Rio Grande do Sul. Para este fim, é apresentado um modelo econométrico espacial para os determinantes da criminalidade. O modelo segue as contribuições da economia do crime, porém acrescenta as contribuições da abordagem ecológica e as teorias do aprendizado social. No modelo, a criminalidade em cidades pode ser explicada por características locais em que o ambiente, a vizinhança e o histórico do indivíduo afetam a criminalidade. São utilizados dados municipais agregados para homicídios, roubos e furtos no ano de 2000. Os testes realizados mostram a existência de dependência espacial em roubos e furtos e independência espacial em homicídios. No artigo ficam destacados os papéis da desigualdade de renda e das aglomerações urbanas como fatores que potencializam a criminalidade em cidades. No artigo são discutidas também as importâncias da família e da escola na explicação da criminalidade. Os resultados obtidos mostram que problemas na estrutura familiar e a ineficiência do ensino no Estado afetam positivamente a criminalidade. Neste artigo, os benefícios do crime e os custos de oportunidade são divididos, o que permite concluir que o crescimento econômico não implica diretamente o aumento da criminalidade. Isto porque se houver um aumento da renda dos mais pobres a criminalidade diminui.

Palavras-chave: Crime; cidades; abordagem ecológica; econometria espacial.

\section{Spatial analysis of criminality in Rio Grande do Sul State, Brazil}

\footnotetext{
Abstract : This paper does a spatial analysis of crime in Rio Grande do Sul. It presents a spatial econometric model for criminality determinants. The model follows main contributions to the economics of crime, but it includes contributions from the ecological approach and social learning. In the model, the criminality in

1 Universidade Federal do Rio Grande do Sul (UFRGS). Doutorando em Economia Aplicada. E-mail: cristiano.oliveira@ufrgs.br.
} 
DE OLIVEIRA, C. Análise espacial da criminalidade no Rio Grande do Sul

cities can be explained by local characteristics, and the environment, the neighborhood and the individual's history affect the criminality. The paper uses aggregate city data for homicides, robberies and thefts in a cross-sectional model for the year of 200o. The findings show the existence of spatial dependence in robberies and thefts and spatial independence in homicides. The paper emphasizes the role of income inequality and of urban agglomerations as factors that increments the criminality in cities. It also discusses the importance of family and schooling on explaining criminality. It shows that problems in the family structure and in schooling affect the crimes positively. The benefits of crime and opportunity costs are separated, which allows concluding that economic growth does not increase criminality. This happens if there is an increase in the income of the poorest, leading to a fall in criminality.

Keywords: Crime; cities; ecological approach; spatial econometrics.

JEL: O10, K42, C31.

\section{Introdução}

O crime tornou-se um tema relevante na sociedade brasileira contemporânea. Esta relevância pode ser justificada pelo fato de o Brasil apresentar índices de criminalidade até cinco vezes maior do que de países europeus. Enquanto na Europa as taxas de homicídios dificilmente superam 5 por 100.000 habitantes ${ }^{2}$, no Brasil segundo o Ministério da Justiça esta taxa é de 23 por 100.000 habitantes. Neste contexto adverso, em que a população brasileira sofre com a violência, a realidade do Rio Grande do Sul não é muito diferente. Se por um lado o Estado apresenta uma das taxas de homicídios mais baixas do Brasil, cerca de 13 por 100.000 habitantes, por outro lado, o Estado é o segundo colocado em furtos, cerca de 2.500 por 100.000 habitantes, e o quarto colocado em roubos, cerca de 650 por 100.000 habitantes. Isto enquanto a média brasileira para furtos é de 1.200 por 100.000 habitantes e para roubos é de 480 por 100.000 habitantes. Estes números de certa forma justificam o porquê de a criminalidade ser muito discutida pelos meios de comunicação e ter sido um dos temas mais discutidos nas ultimas campanhas eleitorais para o dado governo.

Apesar de toda a exposição na mídia e de haver um reconhecimento por parte de autoridades governamentais da necessidade de se entender a dinâmica da criminalidade existem poucos trabalhos científicos sobre o tema. Várias podem ser as causas para esta escassa produção. Em primeiro lugar, a inexistência de estatísticas disponíveis com padronização e confiabilidade. Em segundo lugar, é difícil negar que a criminalidade é um tema complexo, que envolve teorias originadas nas mais variadas

2 Fajnzylber et al. (200o). 
áreas do conhecimento, que vão desde a Sociologia, Psicologia e Criminologia até a Demografia e a Economia.

O primeiro problema tem sido resolvido com a utilização de estatísticas disponibilizadas por algumas secretarias estaduais de segurança pública, uma vez que o Ministério da Justiça só divulga dados estaduais. Isto explica o porquê de muitos trabalhos realizados utilizarem dados estaduais, entretanto a utilização destes dados na forma de médias estaduais podem implicar na generalização de problemas que são localizados. Um breve mapeamento do crime por cidades permite observar que os altos índices de criminalidade atingem apenas algumas regiões de Estado, uma vez que diferentes cidades apresentam diferentes níveis de crime. Mas o que explica tal comportamento? A ciência econômica certamente tem contribuições importantes para a busca desta resposta, uma vez que possui um diferencial metodológico que permite aliar as contribuições teóricas as evidências empíricas. Isto claro, sem negligenciar as importantes contribuições de outras ciências. Este é o enfoque deste artigo: a utilização da metodologia econômica em um contexto multidisciplinar para investigar as causas da criminalidade e sua distribuição espacial no Estado do Rio Grande do Sul.

Este artigo parte do pressuposto que cada cidade possui um ambiente próprio, incluindo a sua vizinhança, que determina seu nível de criminalidade. Esta não é uma idéia tão nova, pois a origem das teorias que incluem o ambiente na explicação da criminalidade são do início do século passado, a partir das contribuições de Shaw e McKay (1929, 1931, 1942). Os autores estudaram o papel das características da vizinhança dos indivíduos no comportamento dos mesmos e concluíram que um ambiente socialmente desorganizado é associado com maiores índices de criminalidade. Os trabalhos feitos por economistas de certa forma sempre incorporaram estes conceitos teóricos, pois geralmente associam os índices de criminalidade a condições socioeconômicas de alguma unidade geográfica. Entretanto, os modelos teóricos econômicos de crime, cuja maioria é baseada exclusivamente no modelo de Becker (1968), associam ao crime um caráter excessivamente econômico. O problema de tomada de decisão dos indivíduos, envolvendo a escolha entre o mercado de trabalho lícito e ilícito, parece ser resolvido no "vácuo".

Este artigo incorpora ao modelo econômico proposto por Becker (1968) as contribuições da abordagem ecológica desenvolvida por Brofenbrenner (1979). A idéia central é mostrar que o ato criminoso surge de um processo em que o papel do histórico do indivíduo e a sua inserção no contexto (ambiente) são fundamentais na explicação do mesmo. Além da utilização da abordagem ecológica de Brofenbrenner, este artigo incorpora as teorias da aprendizagem social. Estas sustentam que crime é algo que se aqprende mediante um complexo processo 
de interação social. Assim, é possível afirmar que crime é algo que se difunde e que a criminalidade de uma cidade pode afetar uma cidade vizinha. Este processo de difusão do crime faz com que exista uma dependência espacial na criminalidade entre cidades e regiões, conforme já foi identificado nos trabalhos de Messner et al. (1999), Messner e Anselin (2001), Puech (2004) e Almeida et al. (2005). Desta forma, modelos econométricos sobre os determinantes da criminalidade devem testar a existência de dependência espacial e corrigi-la quando esta for identificada.

Incorporando estas contribuições teóricas, este artigo faz uma análise espacial da criminalidade no estado. O objetivo é investigar os determinantes da criminalidade e a existência de dependência espacial na criminalidade do estado. Para este fim, o artigo apresenta mais quatro seções. A segunda seção apresenta um arcabouço teórico que visa entender os determinantes da criminalidade, ou seja, quais fatores serão determinantes para que um indivíduo cometa ou não um crime. Nesta seção o enfoque dado ao tema por economistas é reavaliado e complementado por algumas contribuições feitas pela abordagem ecológica proposta por Brofenbrenner (1979) e pelas teorias da aprendizagem social. A terceira seção apresenta um modelo formal, baseado em Fajnzylber et al (2000), que incorpora as teorias propostas. A quarta seção traz a evidência empírica do modelo proposto. Nesta seção é feita uma análise exploratória do crime no estado, com a identificação da existência de dependência espacial e de pontos críticos de criminalidade. A seção apresenta também a implementação empírica do modelo econométrico proposto para as cidades gaúchas no ano de 2000, assim, a teoria e o modelo são associados às variáveis disponíveis. São apresentados as fontes dos dados utilizados, a metodologia de estimação e os respectivos resultados. Estes são discutidos e interpretados à luz das teorias propostas. Ao final do artigo são apresentadas algumas conclusões.

\section{Criminalidade em cidades: abordagem ecológica e a dependência espacial}

A construção de um arcabouço teórico sólido para a explicação das causas da criminalidade em cidades certamente é uma tarefa difícil. Isto porque não são poucas as correntes teóricas distintas que abordam o tema. Na literatura são encontradas diferentes teorias que explicam o fenômeno da criminalidade ${ }^{3}$. A ciência econômica certamente tem uma

3 Ver Molina e Gomes (2002) para uma revisão desta literatura. 
contribuição para este problema social relevante. Entretanto, deve-se ter o cuidado de não dar um caráter excessivamente financista ao tema. Isto porque a decisão de praticar um ato criminoso é um processo complexo em que a história do indivíduo e o seu ambiente influenciam o resultado de sua decisão. A verdade é que a busca por uma explicação mais completa pelos determinantes da criminalidade deve incorporar estes fatores. Desta forma, esta seção visa apresentar alguns argumentos teóricos que serão incorporados no modelo que será apresentado na próxima seção.

Um dos enfoques principais deste artigo é o papel do custo moral como barreira à entrada na atividade criminosa. $\mathrm{O}$ ato de cometer um crime, ou seja, violar uma regra socialmente aceita, pode ou não envolver um custo moral. Mesmo que do ponto de vista financeiro a melhor decisão pareça ser a opção pelo mercado ilícito, a inclusão de um custo moral no modelo pode criar a barreira necessária para a entrada neste mercado. A eficiência do custo moral como barreira à entrada na atividade ilícita é condicionada a um julgamento moral executado pelo indivíduo sobre seu ato. O processo de construção do julgamento moral para os psicólogos desenvolvimentistas ${ }^{4}$ é um processo longo que vai desde a infância do indivíduo até a sua idade adulta. Neste processo, a construção de valores é autoconstruída a partir da história e da cultura que o cerca. Não há uma imposição das regras e normas, mas uma escolha individual de quais devem ou não ser seguidas. Ao longo de sua vida os indivíduos vão construindo relações que começam inicialmente com seus familiares vão até a sua inserção na sociedade na fase adulta. Cada indivíduo se desenvolverá em um determinado contexto histórico. Este contexto (ambiente) afeta a construção do julgamento moral, e por conseqüência, também afeta a decisão de cometer um crime ou não.

Apesar de não estudar especificamente a questão da criminalidade, Brofenbrenner (1979) destacou o papel do ambiente no desenvolvimento do indivíduo em vários aspectos. Esta abordagem, conhecida como ecológica, insere os indivíduos em quatro sistemas concêntricos com suas interconexões. Inicialmente, os indivíduos estão inseridos em um microssistema, que são o conjunto de atividades, papéis e relações interpessoais experenciados pelo indivíduo em um ambiente específico. Esse contexto é constituído por relações face-a-face, que começa com a família e amigos próximos e vão sendo acrescentadas ao longo da vida outras relações, tais como a escola, e suas relações com colegas e professores; e o trabalho e suas relações com seus colegas. Cada relação citada compõe um diferente microssistema em que o indivíduo assume um papel diferente. Este conjunto de microssistemas forma o mesossistema, que de outra forma, é o conjunto de relações construídas

4 Entre os psicólogos desenvolvimentistas, citam-se Piaget, Kohlbert e Tapp. 
ao longo da vida. Existe ainda o exossistema, que é composto pelos ambientes nos quais o indivíduo em desenvolvimento não está presente, mas cujos eventos ocorridos nestes influenciam diretamente o seu desenvolvimento. Por exemplo, acesso ao mercado de trabalho por parte dos seus pais. E por fim, existe o macrossistema, que é o conjunto de todos os sistemas e que envolve desde a situação conjuntural econômica que o indivíduo está inserido até o conjunto de valores compartilhados pela sociedade. Estes irão determinar o conjunto de valores morais que irão ser aceitos ou não pela sociedade e como ela reagirá quando estes forem violados, ou seja, este sistema irá determinar as leis e como estas serão cumpridas. Tal sistema tem influência direta nas formas de relação que ocorrem nos sistemas anteriores.

Estas contribuições esclarecem o papel de cada sistema no processo de construção do julgamento moral e, por conseqüência, na decisão de cometer um crime. Em cada etapa do desenvolvimento moral do indivíduo as suas relações nos diferentes contextos poderão determinar os seus parâmetros do que é certo e do que é errado e, portanto, determinará se haverá um custo moral ou não no ato criminoso. Este custo, que não pode ser medido em termos monetários, certamente existe. A principal contribuição da abordagem ecológica neste caso é de que o ambiente altera o julgamento moral do indivíduo e conseqüentemente altera o custo moral. Quando tratamos de criminalidade em cidades é necessário considerar que a decisão de cometer um crime envolve um processo evolutivo anterior ao momento da decisão em que o ambiente de cada cidade é fundamental neste processo. Cada cidade constitui um macrossistema próprio que afeta não somente o custo moral, mas também os custos de execução. Para Sutherland (1940 apud Molina e Gomes 2002) o crime é algo que se aprende. Segundo o autor (p.375):

a capacidade ou destreza e a motivação necessárias para o delito se aprendem mediante o contato com valores, atitudes, definições e pautas de condutas criminais no curso de normais processos de comunicação e interação do indivíduo com seus semelhantes.

Se o indivíduo possui um acesso muito fácil ao mercado ilícito ou interage com participantes deste mercado, isto diminui o custo de execução e planejamento de um crime. Isto porque a passagem do conhecimento de como planejar, de como executar e dos meios para praticar o crime torna-se mais fácil. Portanto, o processo de interação social ${ }^{5}$ potencializa a difusão da criminalidade. Segundo Cohen e Tita (1999) existem duas formas de difusão da mesma. A primeira é através de contatos diretos entre indivíduos, que ao se deslocarem disseminam o crime através da

5 Argumento também utilizado por Glaeser et al. (1996). 
conquista de novos adeptos. Criam assim redes e organizações que difundem a criminalidade(gangues, quadrilhas, etc). A segunda é através da imitação, que dissemina o crime através da observação. Neste caso, por exemplo, os criminosos observam as oportunidades de retorno em uma região ainda não explorada e praticam crimes semelhantes aos praticados em outras regiões mesmo que não haja um contato direto entre estes criminosos.

Neste processo de difusão da criminalidade as cidades possuem um papel relevante, pois há não só uma disseminação interna, mas também uma externa às cidades. Este processo de difusão evidencia uma dependência espacial na criminalidade entre cidades. Entretanto, as formas como este processo atua e as suas limitações geográficas não são tão claras e são a priori difíceis de medir e identificar. É justamente neste contexto adverso que a econometria espacial ajuda a identificar a existência de dependência espacial na criminalidade de cidades e a extensão da mesma. A próxima seção apresenta um modelo econométrico que incorpora os conceitos aqui discutidos.

\section{Um modelo econométrico espacial para os determinantes da criminalidade}

Nesta seção é apresentado um modelo econométrico espacial para os determinantes da criminalidade. Este modelo tem como ponto de partida um modelo de escolha individual racional proposto por Becker (1968) e segue o processo de agregação proposto por Fajnzylber et al. (2000) com algumas modificações. No modelo, o indivíduo irá cometer crimes se seus benefícios forem maiores que seus custos:

$$
\mathrm{B}>\mathrm{OC}+\mathrm{M}+\mathrm{C}+\mathrm{P}(\mathrm{Pu})
$$

em que B representa os benefícios do crime, OC é o custo de oportunidade, $M$ é o custo moral, $C$ é custo de execução e planejamento do crime e o termo $\mathrm{P}(\mathrm{Pu})$ representa o custo associado a punição $(\mathrm{Pu})$ e sua respectiva probabilidade de ocorrer P. Esta relação dada pela equação (1) pode ser reescrita para que exista um retorno líquido da atividade criminal, $\mathrm{RL}$, tal que:

$$
\mathrm{RL}=\mathrm{B}-\mathrm{OC}+\mathrm{C}+\mathrm{P}(\mathrm{Pu})
$$

Assim, um indivíduo comete um crime $(d=1)$ quando:

$$
R L \geq M \text { ou } \quad B-O C+C+P(P u) \geq M
$$


DE OLIVEIRA, C. Análise espacial da criminalidade no Rio Grande do Sul

Um indivíduo não comete crime $(d=O)$ quando:

$$
R L<M \text { ou } B-O C+C+P(P u)<M
$$

em que $d$ representa a decisão de cometer o crime. As equações (3.1) e (3.2) implicam que o ato de cometer um crime depende de uma comparação feita pelo indivíduo entre o retorno líquido da criminalidade e o custo moral de praticar o ato criminoso. Como já foi salientado anteriormente, o custo moral atua como uma barreira à entrada do indivíduo na atividade criminosa.

A decisão individual de cometer ou não um crime em uma cidade depende de alguns fatores endógenos e exógenos. O modelo assume que existem alguns atributos individuais exógenos, representados por $\mathrm{X}$, provavelmente determinados pela história de vida do individuo, que influenciam o seu custo de oportunidade, seu custo moral, custo de execução e planejamento e o custo associado à punição. Estas variáveis também são influenciadas pelo ambiente em que os indivíduos estão inseridos, estas influências são representadas por Z. Existem também características de cada cidade, que podem ser o seu tamanho, sua forma de organização, sua forma de lidar com criminosos, suas formas de inserção social, entre outras. Estas características que representam o macrossistema de cada local são representadas por Y e influenciam diretamente os benefícios do crime e a probabilidade de ser punido. Estas características endógenas locais também afetam o ambiente em que os indivíduos estão inseridos, pois cada cidade tem um conjunto de características diferentes. Desta forma, a equação (3.1) pode ser escrita de modo que:

$\mathrm{d}=1$ quando

$\mathrm{B}(\mathrm{Y})-\mathrm{OC}(\mathrm{X}, \mathrm{Z}(\mathrm{Y}))+\mathrm{C}(\mathrm{X}, \mathrm{Z}(\mathrm{Y}))+\mathrm{P}(\mathrm{Y}) \mathrm{Pu}(\mathrm{X}, \mathrm{Z}(\mathrm{Y})) \geq \mathrm{M}(\mathrm{X}, \mathrm{Z}(\mathrm{Y}))$

Pode-se ainda reescrever a equação (4) como função de algumas características exógenas e endógenas, que determinam o ato criminoso, assim:

$$
d=f(X, Z, Y)=f(\psi)
$$

Assumindo que tanto a probabilidade de cometer um crime quanto à função $f(\psi)$ são lineares, obtém-se uma regressão para cada indivíduo do tipo:

$d=\psi \beta+\mu$ 
As linearidades assumidas, apesar de arbitrárias, permitem a agregação da equação (6) para cidades. Isto é necessário porque os dados utilizados não são individuais, mas agregados em cidades. Assim, a criminalidade é obtida pela média de crimes de uma cidade i em um determinado ponto no tempo. Desta forma, o modelo a ser estimado de criminalidade é dado por:

$$
D_{i}=\psi_{i} \beta+\mu_{i}
$$

A equação (7) implica que a criminalidade na cidade i depende de algumas características endógenas e exógenas agregadas, que podem ser representadas por algumas variáveis socioeconômicas disponíveis. Neste artigo, considera-se ainda a possibilidade de haver alguma dependência (autocorrelação) espacial na criminalidade. Desta forma, o modelo com dependência espacial pode ser representado por:

$$
D_{i}=\rho W_{1} D_{i}+\psi_{i} \beta+v_{i}
$$

Em que $v_{i}=\lambda W_{2} v_{i}+\xi_{i}$ e $\xi_{i} \sim N\left(0, \sigma^{2} I\right) . \mathrm{W}_{1}$ e $\mathrm{W}_{2}$ são conhecidas como as matrizes de pesos espaciais. Estas podem ser uma relação de

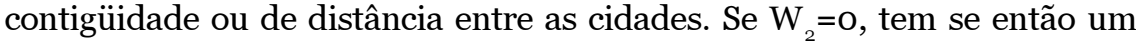
modelo com lag espacial, o que implica que a criminalidade das cidades vizinhas influenciam a criminalidade da cidade i. Isto significa dizer que existe um processo de difusão da criminalidade para toda uma região. $\mathrm{Se} \mathrm{W}_{1}=0$, tem-se um modelo com erro espacial, o que implica que a criminalidade de uma cidade depende de alguma associação espacial de alguma variável explicativa que não foi incluída no modelo. Pode ser o caso de uma variável de difícil mensuração, tal como o acesso a armamentos, a cultura de cada região, religião, etc. Em ambos os casos existem algum tipo de difusão da atividade criminosa. A escolha do modelo mais adequado deve ser baseada em testes estatísticos que serão apresentados adiante.

\section{Uma Evidência Empírica para o Rio Grande do Sul}

Esta seção apresenta a evidência empírica para as cidades do Rio Grande do Sul. Neste artigo analisou-se a criminalidade dos 467 municípios gaúchos no ano de 2000. A escolha do ano de 2000 se deve ao fato de ser este ano o primeiro em que a Secretária Estadual de Justiça e Segurança (SJS-RS) disponibilizou dados municipais sobre criminalidade. 
Desde então, a SJS-RS tem disponibilizado dados mensais. Entretanto, os dados socioeconômicos utilizados neste artigo não possuem a mesma disponibilidade. Assim, a escolha do ano a ser estudado se deve à disponibilidade de dados confiáveis e de boa qualidade fornecidos pelo censo demográfico realizado pelo Instituto Brasileiro de Geografia e Estatística (IBGE). Os roubos são definidos como crimes contra a pessoa, que envolvem algum tipo de ameaça, agressão ou ferimento, e crimes contra o patrimônio. Os furtos são definidos como crimes contra o patrimônio sem a presença das vítimas. A SJS-RS possui 55 categorias para roubos e 22 para furtos. Este artigo utiliza o total de roubos e furtos fornecidos pela SJS-RS. Os dados sobre homicídios são fornecidos pelo DATASUS, órgão ligado ao Ministério da Saúde, e os dados socioeconômicos são fornecidos pelo censo demográfico do IBGE. No apêndice é apresentado um resumo estatístico das variáveis utilizadas.

\subsection{Uma análise exploratória da dependência espacial}

O primeiro passo para a estimação de qualquer modelo espacial é a verificação da existência de alguma relação espacial entre variáveis. Segundo Anselin e Bera (1998) a autocorrelação espacial refere-se à coincidência de valores semelhantes em locais semelhantes. A observação da figura 1 permite deduzir a possibilidade de haver algum tipo de dependência, pois apresenta algumas manchas de cores semelhantes em alguns pontos do Estado em todos os tipos de crime aqui analisados.

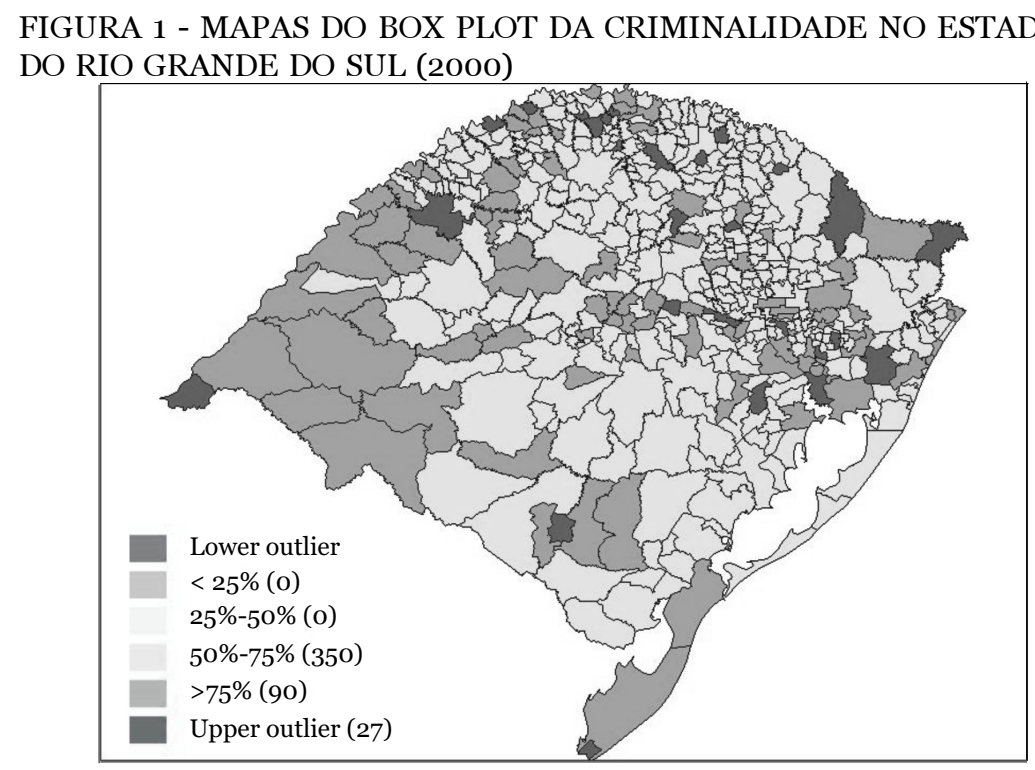


DE OLIVEIRA, C. Análise espacial da criminalidade no Rio Grande do Sul
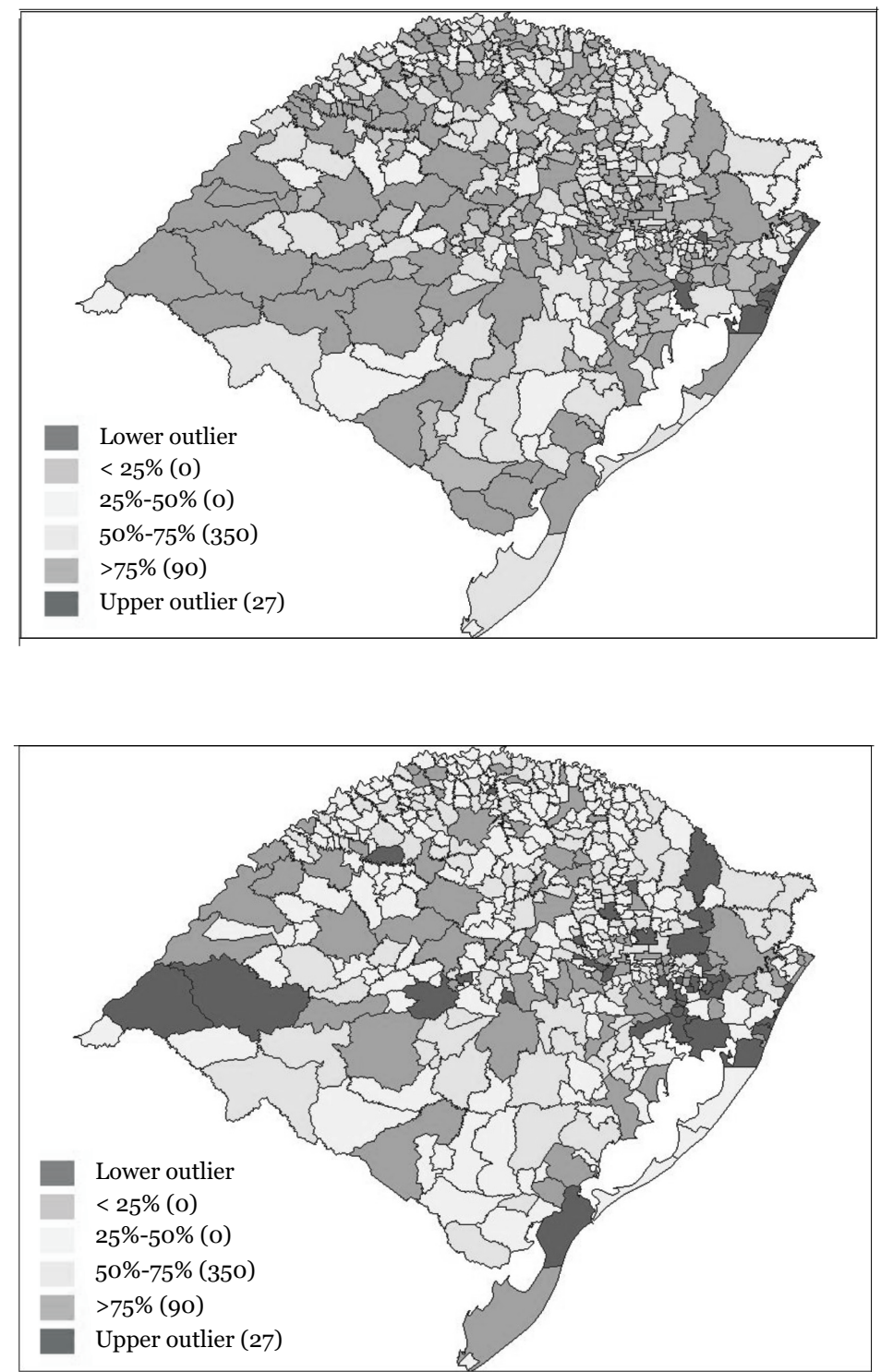

Fonte: Elaborado pelo autor. 
A maneira mais correta de identificar a dependência espacial é através de testes estatísticos. Existem várias formas de testar autocorrelação espacial, mas o teste mais comum é o I de Moran $^{6}$. O teste á dado por:

$$
I=\frac{\sum_{i=1}^{n} \sum_{j=1}^{n} w_{i j} x_{i} x_{j}}{\sum_{i=1}^{n} x_{i}^{2}}
$$

Em que $n$ representa o número de cidades, $w_{i j}$ representa os elementos da matriz de pesos espaciais e $x_{i}$ e $x_{j}$ são os valores da variável analisada em desvios da média. Normalmente estes resultados são muito influenciados pela matriz de pesos espaciais escolhidas. Mas qual matriz deve ser escolhida? A escolha deve ser sempre teórica. Neste artigo a escolha foi por uma matriz de contigüidade "tipo rainha", que considera todas as ordens menores de contigüidade.

A figura 2 mostra um alto valor do I de Moran para roubos (ROUB) e furtos (FURT), mas um baixo valor para homicídios (HOM). O cálculo do I de Moran permite ainda a decomposição das relações espaciais em quatro quadrantes. No primeiro quadrante encontram-se as cidades com alta criminalidade e que possuem alta criminalidade na vizinhança. No terceiro quadrante encontram-se as cidades com baixa criminalidade e baixa criminalidade na vizinhança. Nestes dois quadrantes tem-se o que se chama dependência espacial. Por outro lado, no segundo e quarto quadrantes tem-se alta criminalidade com baixa criminalidade na vizinhança e baixa criminalidade com alta criminalidade na vizinhança e, portanto, existe uma independência espacial.

A identificação das cidades pertencentes ao primeiro quadrante permite localizar as regiões onde há a maior incidência de criminalidade (hot spots) e onde há a maior dependência espacial. A figura 3 mostra estes hot spots e as demais relações para os três tipos de crime.

6 Moran (1948). 
DE OLIVEIRA, C. Análise espacial da criminalidade no Rio Grande do Sul

FIGURA 2 - ESTATÍSTICA GLOBAL DE ASSOCIAÇÃO ESPACIAL (I DE MORAN)
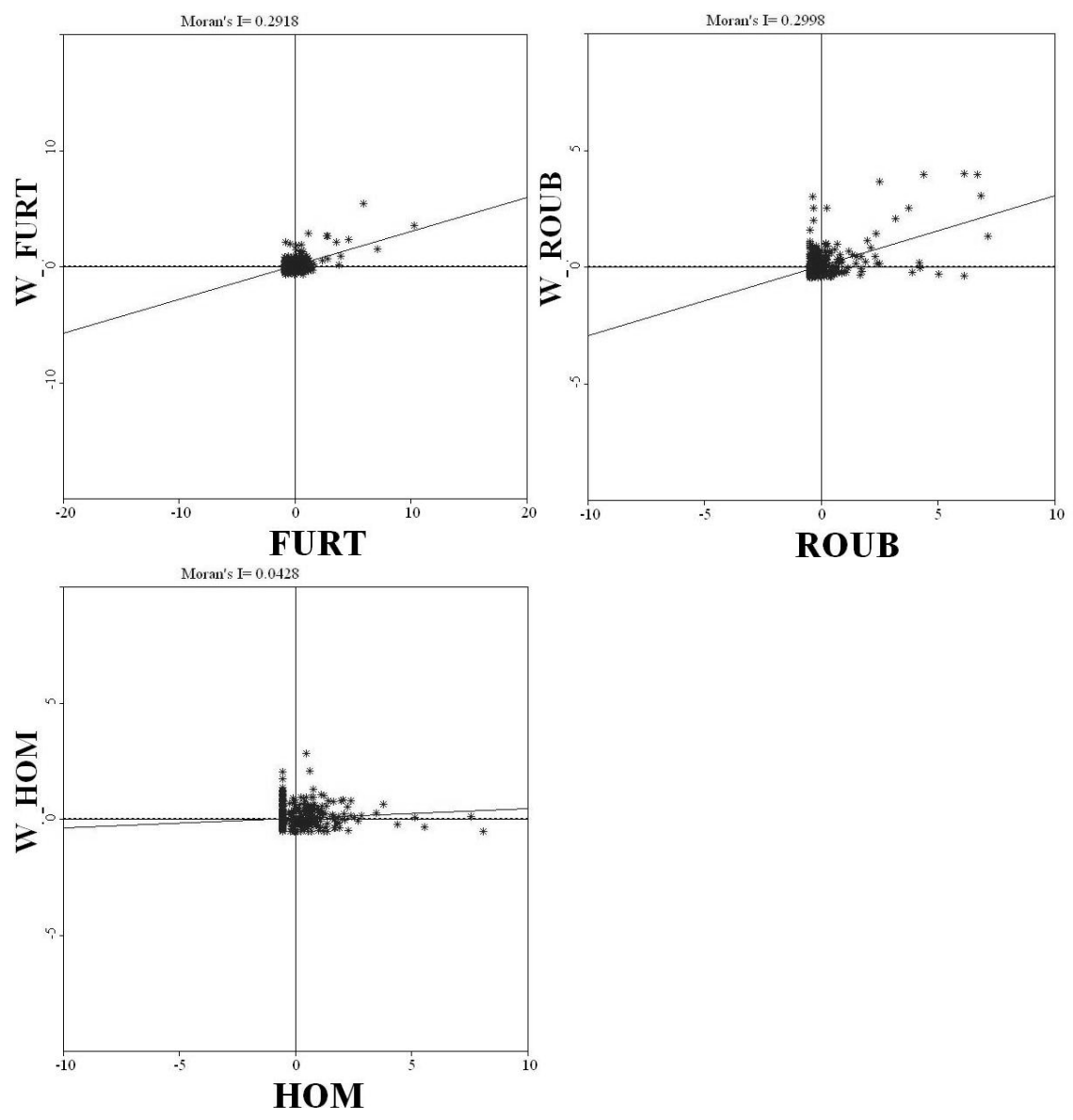

Fonte: Elaborado pelo autor. 
DE OLIVEIRA, C. Análise espacial da criminalidade no Rio Grande do Sul

FIGURA 3 - MAPA DE CLUSTER PARA CRIMES NO ESTADO DO RIO GRANDE DO SUL (2000)
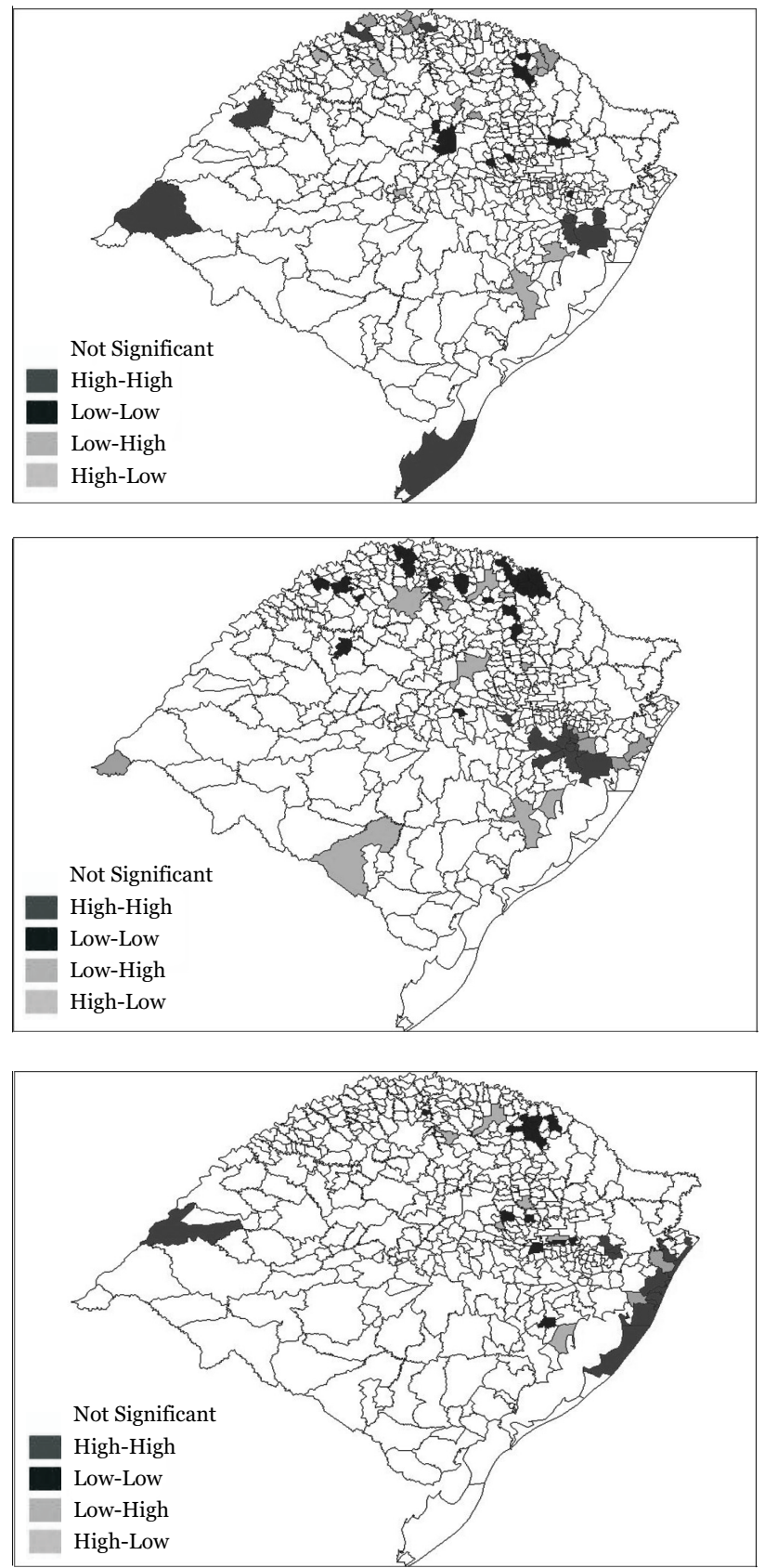

Fonte: Elaborado pelo autor. 
A figura 3 mostra que os homicídios apresentam vários focos espalhados no estado. Existe um foco na região sul, em Santa Vitória do Palmar e Chuí, um na fronteira oeste, próximo a Quaraí e São Borja, um no médio alto Uruguai, próximo a Frederico Westphfalen e outro na região Metropolitana. Este foi o tipo de crime que apresentou a menor dependência espacial e menor concentração. Os roubos apresentam uma concentração na região Metropolitana. Os furtos estão concentrados principalmente no Litoral Norte, onde há um problema histórico com arrombamento de residências que ficam desabitadas na maior parte do ano. É possível observar que há uma forte concentração de crimes na região Metropolitana e seus arredores em pelo menos dois tipos de crimes violentos, homicídios e roubos, o que permite especular a existência de um processo de difusão da criminalidade. Outra consideração possível é que a criminalidade está concentrada nas regiões mais ricas do estado, o que é um forte indício de que o efeito renda é importante na explicação da criminalidade, hipótese esta que será testada no modelo apresentado adiante.

Feita a identificação da existência de dependência espacial, cabe identificar qual modelo econométrico espacial é o mais adequado. Vários testes são possíveis. A tabela 2 apresenta os resultados do modelo econométrico por mínimos quadrados ordinários (MQO) e o teste do multiplicador de Lagrange (LM) para a identificação de ambos os tipos de autocorrelação espacial.

O teste LM avalia a hipótese nula de $\mathrm{r}=\mathrm{O}$ e $\mathrm{l}=\mathrm{O}$ na equação (8) ${ }^{7}$. Este segue uma distribuição $\mathrm{c}^{2} \mathrm{com}$ um grau de liberdade. Vale ressaltar que se houver a rejeição da hipótese nula no modelo com lag espacial, os estimadores de MQO são viesados e ineficientes. Se houver a rejeição da hipótese nula no modelo com erro espacial, os estimadores de MQO são não viesados, mas não são eficientes (Anselin, 1988). Desta forma, os resultados aqui apresentados por MQO devem ser interpretados com cautela, uma vez que os testes indicam a presença de autocorrelação espacial tanto no modelo para roubos quanto para furtos. Já os resultados do teste LM para homicídios mostram a inexistência da dependência espacial e, portanto, a adequação do modelo estimado por MQO. No caso de roubos e furtos, ambos os modelos estimados sugerem um modelo com lag espacial. A próxima seção apresenta os resultados obtidos com os modelos sugeridos pelos testes realizados para cada tipo de crime, bem como a interpretação dos resultados.

7 Ver Anselin e Florax (1995) para uma apresentação formal do teste. 
DE OLIVEIRA, C. Análise espacial da criminalidade no Rio Grande do Sul

TABELA 2 - RESULTADOS POR MÍNIMOS QUADRADOS ORDINÁRIOS

\begin{tabular}{|c|c|c|c|c|c|c|}
\hline & \multicolumn{2}{|c|}{ Homicídios } & \multicolumn{2}{|c|}{ Roubos } & \multicolumn{2}{|c|}{ Furtos } \\
\hline & Eq.(1) & Eq.(2) & Eq.(1) & Eq.(2) & Eq.(1) & Eq.(2) \\
\hline Constante & $\begin{array}{c}3.0590 \\
(2.3102)\end{array}$ & $\begin{array}{l}-23.739 \\
(6.6408)\end{array}$ & $\begin{array}{l}-53.017 \\
(21.559)\end{array}$ & $\begin{array}{l}-474.64 \\
(93.217)\end{array}$ & $\begin{array}{l}-366.49 \\
(112.73)\end{array}$ & $\begin{array}{l}-2663.6 \\
(305 \cdot 34)\end{array}$ \\
\hline Renda1orico & $\begin{array}{c}0.0018 \\
(0.0017)\end{array}$ & & $\begin{array}{c}0.0459 \\
(0.0162)\end{array}$ & & $\begin{array}{c}0.5724 \\
(0.0851)\end{array}$ & \\
\hline Renda2opobre & $\begin{array}{l}-0.0607 \\
(0.0276)\end{array}$ & & $\begin{array}{c}0.1656 \\
(0.2579)\end{array}$ & & $\begin{array}{c}-1.1970 \\
(1.3490)\end{array}$ & \\
\hline $\mathrm{MCF}$ & $\begin{array}{c}1.2433 \\
(0.3979)\end{array}$ & $\begin{array}{c}1.1014 \\
(0.4150)\end{array}$ & $\begin{array}{c}16.235 \\
(3.7140)\end{array}$ & $\begin{array}{c}22.574 \\
(5.8253)\end{array}$ & $\begin{array}{c}137.83 \\
(19.421)\end{array}$ & $\begin{array}{c}86.549 \\
(19.081)\end{array}$ \\
\hline Densidade & $\begin{array}{c}0.0064 \\
(0.0022)\end{array}$ & & $\begin{array}{c}0.4467 \\
(0.0207)\end{array}$ & & $\begin{array}{c}0.2097 \\
(0.1083)\end{array}$ & \\
\hline Habsubnorm & $\begin{array}{c}0.8148 \\
(0.6783)\end{array}$ & & $\begin{array}{c}43.009 \\
(6.3305)\end{array}$ & & $\begin{array}{c}23.024 \\
(33.103)\end{array}$ & \\
\hline Escola & & $\begin{array}{c}2.2433 \\
(0.7546)\end{array}$ & & $\begin{array}{c}113.47 \\
(10.593)\end{array}$ & & $\begin{array}{c}379.52 \\
(34.699)\end{array}$ \\
\hline Gini & & $\begin{array}{c}30.203 \\
(10.953)\end{array}$ & & $\begin{array}{l}-149.12 \\
(153.75)\end{array}$ & & $\begin{array}{c}2093.9 \\
(503.63)\end{array}$ \\
\hline R2 Ajustado & 0.0785 & 0.0734 & 0.6761 & 0.2633 & 0.2470 & 0.3278 \\
\hline AIC & 3716.5 & 3717.1 & 5802.5 & 6184.4 & 7347.6 & 7292.6 \\
\hline $\mathrm{SC}$ & 3741.3 & 3733.7 & 5827.4 & 6201.0 & 7372.5 & 7309.2 \\
\hline LIK & -1852.2 & -1854.5 & -2895.2 & -3088.2 & -3667.8 & -3642.3 \\
\hline $\begin{array}{l}\text { LM robusto } \\
\text { (erro) }\end{array}$ & 1.6825 & 0.2293 & 2.0020 & 4.3361 & 3.8840 & 14.844 \\
\hline $\begin{array}{l}\text { LM robusto } \\
\text { (lag) }\end{array}$ & 1.1218 & 0.4969 & 4.0169 & 5.6627 & 5.0737 & 3.1553 \\
\hline
\end{tabular}

\subsection{Análise dos resultados obtidos pelo modelo econométrico espacial}

A implementação econométrica do modelo teórico apresentado na seção 2 é dividida em dois modelos. A equação (1) apresenta uma equação básica. Nela estão contidas as variáveis tradicionalmente utilizadas na explicação da criminalidade. A equação (2) apresenta duas variáveis muito utilizadas pela literatura na explicação da criminalidade, a escola e a desigualdade de renda. Estas tiveram que ser separadas do modelo básico devido a sua correlação com outras variáveis, principalmente com as variáveis relacionadas à renda. Assim, para evitar um problema 
de multicolinearidade optou-se por estimar dois modelos. Os resultados obtidos já considerando o modelo adequado de autocorrelação espacial são apresentados na tabela 3 em que são apresentados os testes de normalidade dos resíduos proposto por Jarque-Bera. Em todos os modelos a hipótese de não normalidade dos resíduos não pode ser rejeitada ao nível de 1\%. Assim, um método de estimação adequado é o de mínimos quadrados em dois estágios (variáveis instrumentais), uma vez que este método não necessita da hipótese de normalidade dos resíduos para gerar estimadores consistentes.

Cabe ressaltar que a interpretação dos parâmetros estimados no modelo com lag espacial é diferenciada. Quando em (8) tem-se $\mathrm{W}_{2}=0$, o modelo com lag espacial será:

$$
D_{i}=\rho W_{1} D_{i}+\psi_{i} \beta+\xi_{i}
$$

O modelo pode ser reescrito da seguinte forma:

$$
D_{i}=(I-\rho W)^{-1}\left[\psi_{i} \beta+\xi_{i}\right]
$$

O efeito marginal de uma mudança de y em $\mathrm{D}_{\mathrm{i}}$ será:

$$
\frac{\partial D}{\partial \psi}=(I-\rho W)^{-1} \beta
$$

onde o termo (I-rW) ${ }^{-1}$ é chamado de multiplicador espacial (Anselin 2002) que é equivalente a reescrever (11) como a soma de progressão geométrica infinita, da seguinte forma:

$$
\frac{\partial D}{\partial \psi}=I \beta+\rho W \beta+\rho^{2} W^{2} \beta+\rho^{3} W^{3} \beta+\ldots
$$

O primeiro termo desta soma é uma matriz com os efeitos diretos de y em D. O segundo termo representa o efeito da vizinhança, o terceiro termo representa o efeito da vizinhança da vizinhança e assim por diante. Logo, os parâmetros estimados no modelo são globais, uma vez que são considerados todos os efeitos diretos e indiretos das variáveis independentes sobre a variável dependente. No caso deste artigo, em que a variável dependente é a criminalidade, os efeitos indiretos das variáveis independentes representam a atuação das externalidades espaciais. Estas evidenciam o processo de difusão da criminalidade entre cida- 
des. Neste artigo, os modelos para roubos e furtos apresentam este tipo de difusão.

Este resultado é confirmado pelos parâmetros representantes da dependência espacial nos modelos para roubos e furtos, pois eles apresentam resultados positivos e significativos. Vale destacar o bom ajustamento do modelo para roubos. Entretanto, deve ser ressaltado que este $\mathrm{R}^{2}$ não pode ser comparado com os obtidos por MQO, uma vez que o pseudo $\mathrm{R}^{2}$ obtido neste modelo é a razão entre a variância dos valores preditos e a variância dos valores observados para a variável dependente. Apesar de Anselin e Hudak (1992) mostrarem que estas medidas de ajustamento no caso de MQO são equivalentes, no caso de um modelo com lag ou erro espacial isto não é verdadeiro.

A maior parte dos sinais das variáveis incluídas nos modelos são os esperados. A priori espera-se que a renda, que é uma variável endógena, possua um sinal (esperado) ambíguo, pois tem efeitos tanto nos benefícios do crime quanto nos custos de oportunidade. A fim de evitar esta ambigüidade, este artigo decompõe a renda em duas partes: a renda média dos dez por cento mais ricos representa o benefício do crime e a renda média dos vinte por cento mais pobres representa o custo de oportunidade. Os resultados são os esperados: a renda dos mais ricos aumenta os roubos e furtos, mas não afeta os homicídios. Isto se deve a dificuldade de criar uma proxy para o benefício deste tipo de crime, que pode ou não envolver benefícios financeiros. Cabe salientar que nos casos dos modelos com lag espacial, o parâmetro estimado também considera a renda da vizinhança. A renda dos mais pobres afeta negativamente os homicídios, mas os resultados para roubos e furtos são não significativos e ainda apresentam um sinal contrário ao esperado tanto para roubos quanto para furtos.

A densidade demográfica afeta positivamente todos os tipos de crimes. A verdade é que a maior parte dos crimes está concentrada nos ambientes urbanos do estado, apesar de furtos também serem verificados no litoral norte do estado, que não é uma região muito populosa. Os roubos são crimes freqüentes nos ambientes urbanos. Neste caso, a densidade demográfica é uma variável endógena que aumenta os roubos porque o ambiente urbano aumenta os benefícios, diminui a probabilidade de ser punido e diminui custos de execução e planejamento deste tipo de crime. Por exemplo, a densidade demográfica reduz a probabilidade de punição porque existe um anonimato em regiões mais densas. Inicialmente, não existe uma ligação entre a polícia e os residentes, ou seja, diferentemente de uma cidade pequena em que a polícia conhece praticamente toda a população, em cidades grandes os policiais têm dificuldades para identificar criminosos potenciais. Por sua vez, o retorno do crime aumenta e os custos de execução diminuem em regiões densas 
DE OLIVEIRA, C. Análise espacial da criminalidade no Rio Grande do Sul

TABELA 3 - RESULTADOS COM CORREÇÃO PARA A DEPENDÊNCIA ESPACIAL

\begin{tabular}{|c|c|c|c|c|c|c|}
\hline & \multicolumn{2}{|c|}{ Homicídios } & \multicolumn{2}{|l|}{ Roubos } & \multicolumn{2}{|l|}{ Furtos } \\
\hline & Eq.(1) & Eq.(2) & Eq.(1) & Eq.(2) & Eq.(1) & Eq.(2) \\
\hline Constante & $\begin{array}{l}3.0590 \\
(2.3102)\end{array}$ & $\begin{array}{l}-23.739 \\
(6.6408)\end{array}$ & $\begin{array}{l}-60.08^{* *} \\
(22.012)\end{array}$ & $\begin{array}{l}-498.46 \\
(86.938)\end{array}$ & $\begin{array}{l}-639.49 \\
(99.703)\end{array}$ & $\begin{array}{l}-2791.7 \\
(282.25)\end{array}$ \\
\hline Renda1orico & $\begin{array}{l}0.0018 \\
(0.0017)\end{array}$ & & $\begin{array}{l}0.0464^{*} \\
(0.0162)\end{array}$ & & $\begin{array}{l}0.5565^{*} \\
(0.0770)\end{array}$ & \\
\hline Renda2opobre & $\begin{array}{l}-0.0607^{*} \\
(0.0276)\end{array}$ & & $\begin{array}{l}0.1072 \\
(0.2602)\end{array}$ & & $\begin{array}{l}-1.1214 \\
(1.2203)\end{array}$ & \\
\hline MCF & $\begin{array}{l}1.2433 \\
(0.3979)\end{array}$ & $\begin{array}{l}1.1014^{*} \\
(0.4150)\end{array}$ & $\begin{array}{l}15.509^{*} \\
(3.7498)\end{array}$ & $\begin{array}{l}18.755^{*} \\
(5.5539)\end{array}$ & $\begin{array}{l}121.77^{*} \\
(18.770)\end{array}$ & $\begin{array}{l}75.966^{*} \\
(18.354)\end{array}$ \\
\hline Densidade & $\begin{array}{l}0.0064^{*} \\
(0.0022)\end{array}$ & & $\begin{array}{l}0.4172^{*} \\
(0.0254)\end{array}$ & & $\begin{array}{l}0.1703^{* * *} \\
(0.0986)\end{array}$ & \\
\hline Habsubnorm & $\begin{array}{l}0.8148 \\
(0.6783)\end{array}$ & & $\begin{array}{l}42.089^{*} \\
(6.3490)\end{array}$ & & $\begin{array}{l}24.644 \\
(29.849)\end{array}$ & \\
\hline Escola & & $\begin{array}{l}2.2433^{*} \\
(0.7546)\end{array}$ & & $\begin{array}{l}104.49^{*} \\
(10.289)\end{array}$ & & $\begin{array}{l}370.32^{*} \\
(31.949)\end{array}$ \\
\hline Gini & & $\begin{array}{l}\text { 30.203* }^{*} \\
(10.953)\end{array}$ & & $\begin{array}{l}-57.470^{*} \\
(146.17)\end{array}$ & & $\begin{array}{l}2096.07^{*} \\
(462.85)\end{array}$ \\
\hline $\mathbf{p}$ & & & $\begin{array}{l}0.1342^{* *} \\
(0.0671)\end{array}$ & $\begin{array}{l}0.3278^{*} \\
(0.1188)\end{array}$ & 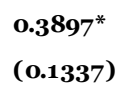 & $\begin{array}{l}0.2863^{* *} \\
(0.1165)\end{array}$ \\
\hline $\begin{array}{l}\text { R2 Ajustado } \\
\text { Jarque-Bera }\end{array}$ & $\begin{array}{l}0.0785 \\
13068.85\end{array}$ & $\begin{array}{l}0.0734 \\
12629.59\end{array}$ & $\begin{array}{l}0.6837 \\
45143.68\end{array}$ & $\begin{array}{l}0.3028 \\
12356.32\end{array}$ & $\begin{array}{l}0.3012 \\
40164.83\end{array}$ & $\begin{array}{l}0.3596 \\
47586.01\end{array}$ \\
\hline
\end{tabular}

Notas: Erros padrão entre parênteses. Significativos a $1 \%(*), 5 \%(* *)$ e $10 \%\left({ }^{* * *}\right)$.

porque nestas regiões o criminoso pode selecionar melhor a sua vítima, dada a grande quantidade de vitimas potenciais. Glaeser \& Sacerdote (1999:12), destacam:

If the method of street criminals is essentially to sit and wait for prospective victims who come within their range of sight, a dense area will have a much larger stream of potential victims then an empty area. 
Neste caso, os criminosos não precisam procurar as vítimas, pois elas vêm até ele. Isto permitiria ao criminoso obter algum tipo de ganho de escala aumentando o seu número de crimes e assim, aumentando o seu retorno da atividade criminosa.

A variável mulheres chefes de família, dada pelo percentual de famílias cuja única fonte de renda é originária de indivíduos do sexo feminino, representa o papel da família no desenvolvimento do indivíduo. Este é um fator exógeno relevante nos determinantes da criminalidade. Outros trabalhos, tais como Araújo Jr e Fajnzylber (2001a,b) e Glaeser e Sacerdote (1999), também incluem esta variável e, assim como este artigo, encontram um sinal positivo para a mesma. A explicação para este resultado é que apesar de famílias monoparentais serem um fenômeno cada vez mais comum, em média elas representam um fator de risco mais do que um fator de proteção com relação à criminalidade. Estas famílias quando chefiadas por mulheres possuem vários problemas que vão desde a redução da renda familiar, pois há somente uma fonte de renda, até problemas para a criação dos filhos, uma vez que a chefe de família é a responsável pelo sustento da residência. Nesses casos não é incomum a criação dos filhos por irmãos mais velhos, por outros familiares e em casos extremos, o seu abandono, o que os força a ir para as ruas.

Estes problemas no microssistema dos indivíduos afetarão o seu desenvolvimento, desenvolvimento esse que inclui a moral. Essas relações farão parte da história de vida do indivíduo e serão partes da construção dos seus valores morais. Estes afetam diretamente os custos morais de se praticar um crime e por conseqüência afetam a sua opção entre o mercado lícito e ilícito. Logo, a presença de famílias monoparentais chefiadas por mulheres representa uma condição de vulnerabilidade que afeta positivamente a criminalidade. No presente estudo ${ }^{8}$, esta variável mostrou-se positiva e significativa em todos os modelos de crime estimados, o que corrobora os argumentos aqui apresentados.

Outro tema controverso é sobre o papel da escola na redução da criminalidade. A priori espera-se que a escola reduza a criminalidade. A explicação econômica é simples um baixo capital humano implica baixos retornos no mercado lícito e isto implica baixos custos de oportunidade para praticar crimes. Outro papel relevante da escola seria a sua participação no processo de desenvolvimento moral do indivíduo, pois nela os indivíduos passam por experiências sociais fora de seu microssistema inicial. Assim, ela tem um papel no processo de inclusão social do indivíduo, quando este passa a interagir com outros indivídu-

8 Assume-se aqui uma estabilidade temporal destes efeitos. 
Esta explicação consideraria a escola como um fator exógeno na explicação da criminalidade em cidades, pois estudar ou não seria uma decisão individual. Entretanto, a escola é também um fator endógeno, pois o acesso ao ensino não depende somente do indivíduo, mas também do contexto em que o mesmo está inserido. Assim, a escola é uma variável que representa tanto fatores exógenos quanto endógenos.

Apesar de o acesso a escola ter uma participação relevante na explicação da criminalidade o que se vê é que a maioria dos trabalhos não utiliza esta variável e quando utiliza encontra resultados insignificantes para a escolaridade. Este é o caso de Glaeser \& Sacerdote (1999) e Araújo Jr e Fajnzylber (2001). Ehrlich (1972) encontrou resultados positivos. Somente Kume (2004) encontra um sinal negativo. Neste artigo, a escola apresenta resultados positivos e significativos para todos os tipos de crime. Uma explicação para este resultado seria que a escola não está conseguindo cumprir seu papel de garantir a inserção no mercado trabalho lícito e nem está conseguindo passar valores a seus alunos. Uma outra explicação é que este resultado se deve a sua alta correlação com a renda dos mais ricos e, portanto, é apenas o resultado de sua relação direta com o benefício do crime.

Outra variável explicativa relevante é a desigualdade de renda. É possível afirmar inclusive que o fato da desigualdade de renda afetar positivamente já pode ser considerado como estilizado uma vez que todos os trabalhos empíricos chegam ao mesmo resultado. Tanto que existem alguns trabalhos somente interessados em explicar esta relação. A explicação mais comum feita por economistas ${ }^{9}$ é de que a desigualdade de renda serve como proxy para a diferença entre os benefícios do crime, representada pela renda das vítimas potenciais, com maior renda e os custos de oportunidade para o crime, representado pela renda de criminosos potenciais, com menores rendimentos. Isto explica o porquê de a equação (2) em todos os tipos de crime excluir as variáveis relacionadas com a renda, pois a desigualdade capta justamente a diferença entre estas variáveis e existe uma alta correlação entre as mesmas. Desta forma, este artigo não apresenta nenhum resultado diferente dos trabalhos anteriores. A desigualdade afeta positivamente todos os tipos de crime.

Estes resultados também podem ser explicados pela perspectiva sociológica, que explica esta relação relevante entre criminalidade e distribuição de renda pela anomia. A frustração de observar determinadas metas que não terão condições de serem atingidas levam o indivíduo a criminalidade por dois meios: Em primeiro lugar, afetando a relação custo de oportunidade e benefício do crime, no caso de o mercado ilícito oferecer uma perspectiva de ganhos futuros maiores do que o lícito,

9 Ver Fajnzylber et al. (2000). 
e em segundo lugar, se isto lhe causar algum tipo de revolta, reduziria o seu custo moral de praticar um crime. Reduziria estes custos porque o indivíduo não se sentindo parte daquele ambiente experimentado por outros, se sente injustiçado e pratica o crime a fim de restabelecer a "justiça social". Este fenômeno sociológico certamente esta associado às características da cidade, o que implica tratar-se de uma variável endógena.

A variável habitações subnormais representa o percentual de indivíduos que vivem em habitações consideradas pelo IBGE como em condições inapropriadas para moradia. Esta serve de proxy para ambientes ruins, que incluem vários indivíduos excluídos econômica e socialmente. Estes ambientes ruins podem gerar valores morais distorcidos ${ }^{10}$, que reduziriam os custos morais do crime e poderiam gerar um conhecimento comum da sua prática, que reduziriam os seus custos de execução e de planejamento. Esta é uma variável endógena que deve ser testada, principalmente quando se utilizam dados para cidades, como é o caso do presente artigo. Os resultados obtidos mostram um efeito positivo para roubos, conforme o esperado pela teoria, mas valores insignificantes para homicídios e furtos.

\section{Considerações Finais}

Este artigo teve como objetivo fazer uma análise espacial da criminalidade no Estado do Rio Grande do Sul. Foram identificados geograficamente os locais com altos índices de criminalidade e alta dependência espacial no estado. Considerando esta dependência, o artigo investigou as causas da criminalidade nas cidades gaúchas. Esta investigação levou à conclusão de que a decisão de cometer um crime envolve um processo evolutivo anterior para o qual o ambiente de cada cidade é fundamental. Esse ambiente chamado de macrossistema possui características próprias e transcende as fronteiras municipais, pois existe uma dependência espacial entre cidades. Essa dependência surge do processo de interações sociais que leva à difusão da criminalidade.

O modelo empírico apresentou os resultados esperados pelo modelo teórico, o que reafirma a capacidade da economia não somente de contribuir para a explicação da criminalidade, mas também de sugerir políticas públicas mais eficientes. Se por um lado a utilização da econometria espacial permite a utilização de informações georeferenciadas, permitindo a identificação de regiões que devem ser prioritárias em cada tipo de crime, por outro lado fica evidente pelos argumentos apresentados

10 Este comportamento anti-social é conhecido como subculturas. Ver Mendonça \& Molina (2002) para uma resenha bibliográfica sobre as teorias subculturais. 
no artigo que políticas públicas devem ser globais, pois como a vizinhança importa, uma política pública adotada em uma cidade não reduziria sua criminalidade se os vizinhos não adotassem políticas semelhantes.

No artigo ficou destacado o papel da desigualdade de renda como fator que potencializa a criminalidade. $\mathrm{O}$ aumento da renda dos mais ricos aumenta os roubos e furtos e o aumento da renda dos mais pobres reduz os homicídios. Isto permite concluir que o crescimento da renda não aumenta a criminalidade conforme concluem trabalhos anteriores. Pois segundo o modelo estimado, isto só ocorre quando o crescimento da renda não atinge os mais pobres.

Os resultados obtidos mostraram que o papel da escola na redução da criminalidade não foi o esperado. Os resultados positivos encontrados servem de alerta para o fato de que a escola pode não estar cumprindo com seu papel de inserir o indivíduo no mercado de trabalho e de passar valores morais aos mesmos. Novas pesquisas sobre o tema devem ser feitas a fim de sugerir políticas públicas que garantam que a escola cumpra ambos os papéis.

Ficou destacado também neste artigo o papel da família na explicação da criminalidade. O modelo teórico mostrou que a família tem um papel fundamental na formação de valores morais dos indivíduos, que por sua vez, afetam os custos morais de cometer um crime. Qualquer alteração na estrutura da família pode alterar estes custos e potencializar a criminalidade. Os resultados positivos obtidos para mulheres chefes de família em todos os tipos de crime corroboraram com estes argumentos. Neste caso, políticas públicas tais como programas de planejamento familiar e de redução de gravidez na adolescência seriam as sugestões de política mais recomendadas.

\section{Referências}

ABREU, M. \& DE GROOT, H. \& FLORAX, R. (2004). "Space and Growth." Tinbergen Institute Discussion Paper, TI 2004-129/3.

ALMEIDA E. \& HADDAD, E. \& HEWINGS, G. (2005). “The spatial pattern of crime in Minas Gerais: An exploratory analysis". Economia Aplicada 9: 39-55.

ANSELIN, L. (1988). Spatial Econometrics: Methods and Models. Dordrecht: Kluwer.

ANSELIN, L. (1992). Spacestat tutorial: A workbook for using Spacestat in the analysis of spatial data. Urbana Champaign Illinois.

ANSELIN, L. (2001). "Spatial Econometrics", In: BALTAGI, B. (ed.). A Companion to Theoretical Econometrics. Oxford: Blackwell Publisher. 
DE OLIVEIRA, C. Análise espacial da criminalidade no Rio Grande do Sul

ANSELIN, L. (2002). "Under the hood. Issues in the specification and interpretation of spatial regression models". Agricultural Economics 27: p. $247-267$.

ANSELIN, L. (2003). "Spatial Externalities, Spatial Multipliers and Spatial Econometrics.” International Regional Science Review 26: 153-166.

ANSELIN, L. \& BERA, A. (1998). "Spatial Dependence in Linear Regression Models with an Introduction to Spatial Econometrics", In: ULLAH, A. \& GILES, D (eds), Handbook of Applied Economic Statistics. Berlin: Springer.

ANSELIN, L. \& FLORAX, R. (1995). "Small sample properties of tests for spatial dependence in regression models: Some further results." In: ANSELIN, L. \& FLORAX, R. (eds) New Directions in Spatial Econometrics. Berlin: Springer-Verlag, pp. 21-74.

ARAÚJO, A. \& FAJNZYLBER, P. (2001a). "O que causa a criminalidade violenta no Brasil? Uma análise a partir do modelo econômico do crime: 1981 a 1996". Mimeo.

ARAÚJO, A. \& FAJNZYLBER, P. (2001b). "Violência e Criminalidade" In: LISBOA, M. \& MENEZES-FILHO, N. (eds). Microeconomia e Sociedade no Brasil. Rio de Janeiro: Contracapa.

BECKER, G. (1968). "Crime and Punishment: An Economic Approach." Journal of Political Economy 76: 169-217.

BRONFENBRENNER, U. (1996) [1979]. A ecologia do desenvolvimento humano: experimentos naturais e planejados. Porto Alegre: Artes Médicas.

CERQUEIRA, D. \& LOBÃO, W. (2003a). "Condições socioeconômicas, polícia e produção criminal”. IPEA, Mimeo.

CERQUEIRA, D. \& LOBÃO, W. (2003b). "Determinantes da Criminalidade: Uma Resenha dos Modelos Teóricos e Resultados Empíricos.” IPEA, Mimeo.

COHEN, J. \& TITA, G. (1999). "Spatial Diffusion in Homicide: Exploring a General Method of Detecting Spatial Diffusion Processes." Journal of Quantitative Criminology 15(4): 451-493.

EHRLICH, I. (1972). "The Deterrent Effect of Criminal Law Enforcement." Journal of Legal Studies 1: 259-276.

EHRLICH, I. (1973). "Participation in Illegitimate Activities: A Theoretical and Empirical Investigation." Journal of Political Economy 81: 521-565.

EIDE, E. (1994). Economics of Crime: Deterrence and the Rational Offender, Contributions to Economic Analysis. Amsterdam, Oxford \& Tokyo: NorthHolland.

EIDE, E. (1995). "Economics of Criminal Behavior" Mimeo. URL: http:// encyclo.findlaw.com/810obook.pdf. Acesso em 11 jan. 2005.

FAJNZYLBER, P. \& LEDERMAN, D. \& LOAYZA, N. (1999a). "Inequality and Violent Crime" World Bank, Washington, DC. Mimeo.

FAJNZYLBER, P. \& LEDERMAN, D. \& LOAYZA, N. (1999b). "Inequality and Violent Crime" World Bank, Washington, DC. Mimeo. 
FAJNZYLBER, P. \& LEDERMAN, D. \& LOAYZA, N. (2000). "What Causes Violent Crime." European Economic Review 46: 1323-1357.

FREEMAN, S. \& GROGGER, J. \& SONSTELIE, J. (1996). "The Spatial Concentration of Crime." Journal of Urban Economics 40: 216-231.

GLAESER, E. (1994). “Cities, Information, and Economic Growth”. Cityscape: Journal of Policy Development and Research 1(1):9-47.

GLAESER, E. (1999). “An Overview of Crime and Punishment” Washington: World Bank. Mimeo.

GLAESER, E. \& SACERDOTE, B. \& SCHEINKMAN, J. (1996). "Crime and Social Interactions." Quarterly Journal of Economics 111: 507-548.

GLAESER, E. \& SACERDOTE, B. (1999). "Why is There More Crime in Cities." Journal of Political Economy 107(6): 225-258.

GUTIERREZ, M. B. \& MENDONÇA, M. J. \& SACHSIDA, A. \& LOUREIRO, P. (2004). "Inequality and Criminality Revisited: further evidence from Brazil." Apresentado no encontro nacional da ANPEC 2004.

KUME, L. (2004). "Uma estimativa dos determinantes da taxa de criminalidade brasileira: uma aplicação em painel dinâmico". Apresentado no encontro nacional da ANPEC 2004.

MENDONÇA, M. J. (2001). "Um modelo de criminalidade para o caso brasileiro". Apresentado no encontro nacional da ANPEC 2001.

MESSNER, S. \& ANSELIN, L. (2002). "Spatial analyses of Homicide with Areal Data”. Mimeo. University of Illinois. Urbana- Champaign.

MOLINA, A. \& GOMES, L. (2002). Criminologia. $4^{\mathrm{a}}$ ed. São Paulo: Revista dos Tribunais.

PUECH, F. (2004). "How do Criminals Locate? Crime and Spatial Dependence in Minas Gerais”. Mimeo. CERDI. Auvergne University.

Recebido em: 23 de janeiro de 2007 Primeira resposta em: 10 de maio de 2007 Aceite em: 24 de agosto de 2007 
DE OLIVEIRA, C. Análise espacial da criminalidade no Rio Grande do Sul

\section{Apêndice}

TABELA A.1. - ESTATÍSTICA DESCRITIVA DAS VARIÁVEIS UTILIZADAS

\begin{tabular}{lccccc}
\hline \multicolumn{1}{c}{ Variável } & Observações & Média & $\begin{array}{c}\text { Desvio } \\
\text { Padrão }\end{array}$ & $\begin{array}{c}\text { Míni- } \\
\text { mo }\end{array}$ & Máximo \\
\hline Homicídios & 273 & 9.30 & 11.96 & 0.00 & 108.68 \\
Roubos & 273 & 150.70 & 249.00 & 0.00 & 1610.98 \\
Furtos & 273 & 944.37 & 807.64 & 0.00 & 8111.42 \\
Renda1orico & 273 & 1102.54 & 375.92 & 409.60 & 3268.16 \\
Renda2opobre & 273 & 44.46 & 22.92 & 6.52 & 128.70 \\
MCF & 273 & 4.02 & 1.52 & 0.63 & 10.70 \\
Densidade & 273 & 125.13 & 392.95 & 2.99 & 2900.29 \\
Habsubnorm & 273 & 0.31 & 1.31 & 0.00 & 10.56 \\
Escola & 273 & 5.37 & 0.87 & 3.27 & 8.96 \\
Gini & 273 & 0.53 & 0.06 & 0.36 & 0.80 \\
\hline
\end{tabular}

